Check for updates

Cite this: RSC Adv., 2017, 7, 52337

Received 30th September 2017 Accepted 30th October 2017

DOI: $10.1039 / c 7 r a 10809 e$

rsc.li/rsc-advances

\title{
Comparison of the characteristic properties of reduced graphene oxides synthesized from natural graphites with different graphitization degrees
}

\begin{abstract}
Xuan Jiao, (D) ab Yangshuai Qiu, ${ }^{\text {*ab }}$ Lingyan Zhang $^{\text {ab }}$ and Xudong Zhang ${ }^{\text {ab }}$
In this study, an investigation on the characterization of reduced graphene oxides ( $r G O s$ ) prepared from natural graphites with different graphitization degrees using Hummers method was conducted. X-ray diffraction, X-ray photoelectron spectroscopy, Fourier transform infrared spectroscopy, Raman spectroscopy, atomic force microscopy and electrochemical performance were performed to characterize the as-prepared graphene oxides (GOs) and rGOs. The results demonstrate that graphites with a lower graphitization degree are more easily oxidized due to the active carbon atoms exposed on their edges and the effective diffusion of oxidants that intercalate into the graphitic layers. In addition, graphites with a low graphitization degree were more suitable for the synthesis of thin layer graphene with a high defect degree and small size in the in-plane $\mathrm{sp}^{2}$ domains as well as a relatively high specific capacitance.
\end{abstract}

\section{Introduction}

Since the discovery of graphene in 2004, two-dimensional graphitic material containing hexagonal networks comprised of carbon atoms have gradually gained increasing amounts of attention and research efforts for industrial applications worldwide. ${ }^{1,2}$ Graphene is extensively applied in electronics, energy storage, photovoltaic devices, membrane materials, resources recovery, biological engineering, biocatalysis, original filtration, and lightweight/strong composite materials, attributing to its superior properties, such as outstanding electrical conductivity, high optical transparency, favorable adsorption performances, high thermal conductivity and mechanical strength. $^{3-7}$ With the development of advanced technology, several useful methods have been proposed to synthesize graphene, including mechanical exfoliation, ${ }^{8}$ liquid-phase exfoliation, ${ }^{9}$ bottom-up assembly, ${ }^{10}$ epitaxial growth via thermal graphitization of silicon carbide, ${ }^{11}$ unzipping carbon nanotubes, ${ }^{12}$ epitaxial growth of graphene via chemical vapor deposition (CVD) on substrates ${ }^{13,14}$ and the reduction of graphene oxide. ${ }^{15,16}$ Among these methods, chemical reduction in wet conditions using graphene oxide has been considered as a costeffective and optimized approach for the large-scale production of graphene materials. ${ }^{17}$

Graphite oxide (GrO) is regarded as a superior precursor for the synthesis of graphene and the electrochemical reduction

${ }^{a}$ School of Resources and Environmental Engineering, Wuhan University of Technology, Luoshi Road 122, Wuhan, Hubei 430070, China.E-mail: liucanviva@whut.edu.cn

${ }^{b}$ Hubei Province Key Laboratory for Processing of Mineral Resources and Environment, Wuhan University of Technology, Luoshi Road 122, Wuhan, Hubei 430070, China and chemical reduction methods have been demonstrated to be efficient and productive methods. ${ }^{18,19}$ During the oxidation and reduction processes with the exfoliation of $\mathrm{GrO}$, graphene oxide (GO) and reduced graphene oxide ( $\mathrm{rGO}$ ) are generated. Both $\mathrm{GO}$ and rGO are graphene derivatives that have structures containing defects due to the oxygen-containing functional groups exposed on the edges of the carbon layers. It is evident that numerous variable factors could affect the structure of graphene and the properties of graphene products notably depend on the structure. ${ }^{20}$ As previously reported in the literature, the graphite used as starting material could directly influence the structure and properties, hence, the selectivity of the raw material plays an important role in the preparation of graphene.

Due to its abundant reserves worldwide, graphene is usually prepared from the natural graphite. It has been reported that the crystallinity of the graphite, used as the starting material, can strongly influence the graphene layers formed in the chemical reduction process. ${ }^{21}$ In general, natural graphite can be classified into crystalline graphite and aphanitic graphite. On the basis of the different crystalline morphologies, as well as the presence of oxygen-containing groups on the edges of the structural layers, there is a remarkable difference on the graphitization degree of natural graphites, which can be used to evaluate the development of the graphitic structure in various carbon materials. ${ }^{22-25}$ The degree of graphitization, as a rule, is the extent to which a material approaches the ideal graphite structure, which can be distinguished using XRD and theoretical calculations based on the values of $d_{002}$. As reported in the literature, numerous factors were found to affect the characteristics of rGO, such as the lateral size ${ }^{26}$ impurity content, ${ }^{20}$ geographical origin $^{27}$ and various synthesis methods. ${ }^{28}$ 
However, the concept of graphitization degree of natural graphite has recently attracted attention in scientific research as well as its connection with the characteristic properties of graphitic derivatives. In addition, few studies have investigated the effects of the graphitization degree of graphite used as raw material on the characteristics and properties of rGO prepared from these materials.

Therefore, natural graphites with different graphitization degree was used to prepare GOs and rGOs using the chemical reduction method. In this study, the objective was to focus on the effect of the different graphitization degree in four types of natural graphites on the rGO synthesized from these materials whilst comparing their characteristics and properties.

\section{Experimental}

\subsection{Materials and chemicals}

Samples of natural graphites with different graphitization degree were classified into four types, marked as VG, LG, FG and $\mathrm{AG}$, and used as the starting materials to prepare rGO. In addition, the natural graphite were obtained by screening with the size fraction at $38-45 \mathrm{~mm}$, whose fixed carbon contents exceeded 99\%. Potassium permanganate, sodium nitrate, 98\% sulfuric acid, 36\% hydrochloric acid, 30\% hydrogen peroxide and $85 \%$ hydrazine hydrate were all purchased from Sinopharm Chemical Reagent Co., Ltd, China. All the regents were of analytical grade, used without further purification. Deionized water with a resistivity of $18.25 \mathrm{M} \Omega \mathrm{cm}$ was used in this work and was produced using a Milli-Q Direct 16 purification system.

\subsection{Preparation of GO and rGO}

GrO samples were prepared as reported previously using Hummers' method. ${ }^{29}$ First of all, $3.0 \mathrm{~g}$ of natural graphite and $1.5 \mathrm{~g}$ of $\mathrm{NaNO}_{3}$ were added to $69 \mathrm{~mL}$ of $\mathrm{H}_{2} \mathrm{SO}_{4}$ and stirred using a magnetic stirrer and cooled using an ice-water bath. Next, $9.0 \mathrm{~g}$ of $\mathrm{KMnO}_{4}$ was added slowly with continuous stirring keeping the temperature less than $10^{\circ} \mathrm{C}$. Then, the temperature was allowed to increase to $37^{\circ} \mathrm{C}$ for $2 \mathrm{~h}$. After that, $138 \mathrm{~mL}$ of deionized water was added dropwise to the mixture at $96^{\circ} \mathrm{C}$ and stirred for $30 \mathrm{~min}$. Then, $420 \mathrm{~mL}$ of deionized water was added to dilute the mixture as well as $30 \mathrm{~mL}$ of and aqueous solution of $\mathrm{H}_{2} \mathrm{O}_{2}$ to remove the residual $\mathrm{KMnO}_{4}$. Finally, the mixture was washed with $5 \% \mathrm{HCl}$ aqueous solution and deionized water while centrifuging at $2500 \mathrm{rpm}$ for $30 \mathrm{~min}$. The GrO was washed without $\mathrm{SO}_{4}{ }^{2-}$ and dried at $60{ }^{\circ} \mathrm{C}$ for $24 \mathrm{~h}$ in vacuo.

The as-prepared GrO obtained from the natural graphites with different graphitization degrees were labeled as GrO-VG, GrO-LG, GrO-FG and GrO-AG. After milling the GrOs into powder, the samples were dispersed in deionized water to form yellow-brown suspensions. Next, the suspensions were ultrasonically exfoliated using a Cole Parmer ultrasonic processor with $60 \%$ amplitude for $12 \mathrm{~min}$. Then, the suspensions were centrifuged at $2800 \mathrm{rpm}$ for $20 \mathrm{~min}$ to remove the non-exfoliated GrO. GOs in the supernatants obtained from GrO-VG, GrO-LG, GrO-FG and GrO-AG, and were labeled as GO-VG, GO-LG, GOFG, and GO-AG, respectively.
rGO samples were obtained through the reduction of the asprepared GO supernatants. At first, $5 \mathrm{~mL}$ of $85 \%$ hydrazine hydrate was added to the suspension, while the $\mathrm{pH}$ was adjusted to 10 using a $0.1 \mathrm{M} \mathrm{NaOH}$ aqueous solution. Subsequently, the reaction mixture was kept at $97{ }^{\circ} \mathrm{C}$ for $8 \mathrm{~h}$, then filtered and washed with methanol and water for several times. The rGO powders were obtained after drying at $60{ }^{\circ} \mathrm{C}$ for $24 \mathrm{~h}$ in vacuo. The rGOs obtained after the reduction of GO-VG, GO-LG, GO-FG and GO-AG were labelled as rGO-VG, rGO-LG, rGO-FG and rGOAG, respectively.

\subsection{Measurements}

$\mathrm{X}$-ray diffraction (XRD) patterns were recorded in the range of $2 \theta=5-80^{\circ}$ using a D8 Advance model X-ray powder diffractometer (Bruker Corporation, Stuttgart, Germany) with $\mathrm{Cu} \mathrm{K} \alpha$ radiation $(\lambda=1.5406 \AA)$.

X-ray photoelectron spectroscopy (XPS) patterns were obtained on a VG Multilab 2000 (Thermo Electron Corporation, Waltham, MA, USA) with a monochromatic Al X-ray source operating at $300 \mathrm{~W}$. The spectra were corrected using the $\mathrm{C} 1 \mathrm{~s}$ peak at $284.6 \mathrm{eV}$.

The Fourier transform infrared (FT-IR) spectra within the range from 400 to $4000 \mathrm{~cm}^{-1}$ were recorded on a Nicolet IS-10 infrared spectrometer (Nicolet Corporation, Madison, USA).

The Raman spectra of all the samples were obtained using a Renishaw INVIA Raman microscope (Renishaw Corporation, Gloucestershire, England) equipped with an Ar laser probe at the $514.5 \mathrm{~nm}$ mode, recorded in the Raman region from 500 to $3500 \mathrm{~cm}^{-1}$.

Atomic force microscopy (AFM) images of the as-prepared rGOs were recorded using a Bruker MultiMode 8 AFM (Bruker Corporation, Stuttgart, Germany) in peak force tapping mode.

Cyclic voltammograms (CV) were measured using a $\mathrm{CHI}$ $660 \mathrm{E}$ electrochemical workstation (Shanghai chenghua instrument Co. Ltd, Shanghai, China) in a three-electrode configuration at ambient temperature, in which the rGO-modified glassy carbon electrode, a saturated calomel electrode and a slice of platinum served as the working electrode, reference electrode and auxiliary electrode, respectively. In addition, a $6 \mathrm{~mol} \mathrm{~L}^{-1}$ $\mathrm{KOH}$ aqueous solution was used as the electrolyte. The CV tests were conducted in the sweep potential range from -0.9 to $0.1 \mathrm{~V}$ at a scan rate of $10 \mathrm{mV} \mathrm{s}^{-1}$. The specific capacitance of the electrodes could be calculated using the following equation:

$$
C=\frac{1}{2 \times m \times \Delta V \times \nu} \times\left(\int_{-0.9}^{0.1} I \mathrm{~d} V+\int_{0.1}^{-0.9} I \mathrm{~d} V\right)
$$

where $C$ is the specific capacitance $\left(\mathrm{F} \mathrm{g}^{-1}\right)$ of the as-prepared rGO samples, $I$ is the current response (A) in a potential, $\Delta V$ (V) is the potential window, $\nu\left(\mathrm{mV} \mathrm{s}^{-1}\right)$ is the potential scan rate and $m(\mathrm{~g})$ is the mass of the electroactive materials.

\section{Results and discussion}

\subsection{Characterization of the various graphites}

The XRD patterns of the four natural graphites with different graphitization degrees are shown in Fig. 1. All the patterns 


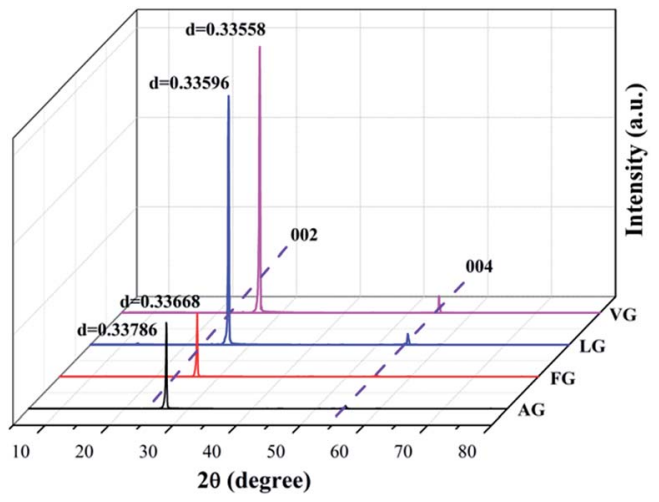

Fig. 1 XRD spectra obtained for VG, LG, FG, and AG.

exhibited strong and sharp peaks at approximately $2 \theta=26.45^{\circ}$ and other peaks with relatively weak intensity appearing at around $2 \theta=54.61^{\circ}$, corresponding to the (002) and (004) reflection planes of natural graphite, respectively. The degree of graphitization for the starting graphite samples can be calculated based on the following equation: ${ }^{30-32}$

$$
g(\%)=\frac{0.3440-d_{002}}{0.3440-0.3354} \times 100
$$

where $g(\%)$ is the degree of graphitization of the natural graphites, 0.3440 is the interlayer spacing of fully nongraphitized carbon ( $\mathrm{nm}$ ) and 0.3354 is the interlayer spacing of an ideal graphite crystallite $(\mathrm{nm})$. The interlayer spacing $\left(d_{002}\right)$ can be obtained from the XRD patterns using the Bragg equation.

The results for the degree of graphitization were $97.91 \%$ for VG, $93.48 \%$ for LG, $85.12 \%$ for FG and $71.39 \%$ for AG, indicating that there was a increasing trend in the crystalline morphology against ideal graphite from AG to VG. In addition, it was obvious that graphite with a high degree of graphitization presented peaks with strong intensity in the XRD spectrum, which indicates that the graphite possessed a superior crystal structure and thereby better diffraction.

The Raman spectra of the natural graphites samples are shown in Fig. 2. The spectrum obtained for each sample showed

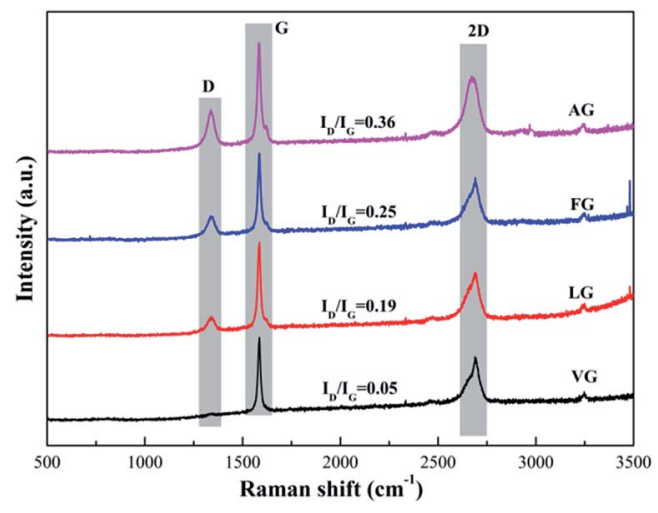

Fig. 2 Raman spectra obtained for VG, LG, FG, and AG. the presence of $\mathrm{D}, \mathrm{G}$ and $2 \mathrm{D}$ bands, located at approximately 1345,1580 and $2670 \mathrm{~cm}^{-1}$, respectively. In addition, the lattice disorder of the graphite derivatives was usually evaluated by the ratio of the intensity of the $\mathrm{D}$ band to the $\mathrm{G}$ band $\left(I_{\mathrm{D}} / I_{\mathrm{G}}\right) \cdot{ }^{33} \mathrm{In}$ addition, the values were determined to be 0.05 for VG, 0.19 for LG, 0.25 for FG and 0.36 for AG, implying that the degree of defects in the samples diminished in the order of the enhancement in the degree of graphitization.

The XPS spectra of the different graphite powders are shown in Fig. 3. Only two intense and distinct peaks at around 283.8 and $531.3 \mathrm{eV}$ were observed, which were attributed to the high

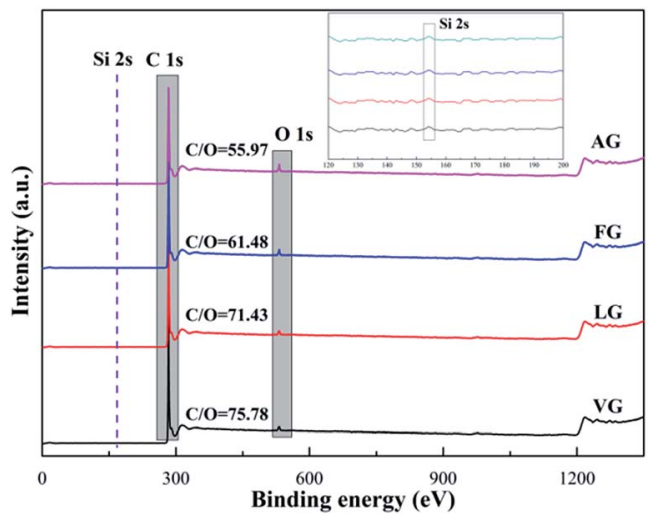

Fig. 3 XPS spectra obtained for VG, LG, FG, and AG.
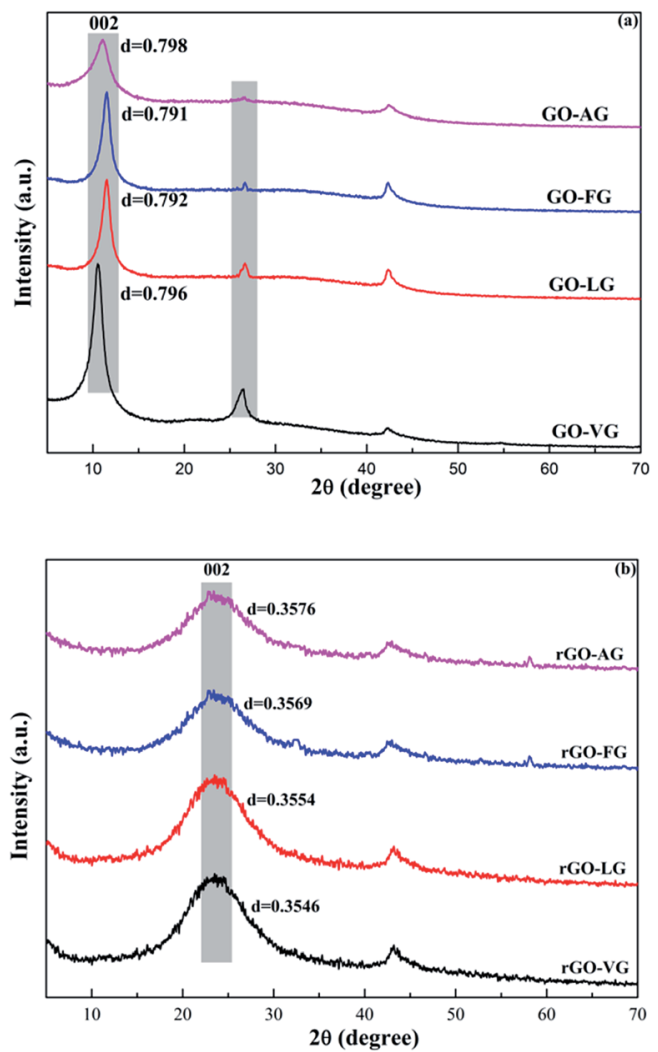

Fig. 4 XRD patterns obtained for the as-prepared GOs (a) and rGOs (b). 
purity of the graphite, corresponding to the $\mathrm{C} 1 \mathrm{~s}$ and $\mathrm{O} 1 \mathrm{~s}$ peaks, respectively. ${ }^{34}$ Furthermore, the other weak peaks at approximately $151.1 \mathrm{eV}$ could be related to $\mathrm{Si} 2 \mathrm{~s}$ caused by a tiny amount of impurity. ${ }^{35}$ On the basis of the results obtained via XPS for the surface atomic concentration, the mass ratio of $\mathrm{C}$ and $\mathrm{O}$ could be obtained and reflected the existence of other oxides. In addition, the values of $\mathrm{C} / \mathrm{O}$ were 75.78 for $\mathrm{VG}, 71.43$ for LG, 61.48 for FG and 55.97 for AG, suggesting that a higher value of $\mathrm{C} / \mathrm{O}$ represented less oxygen-containing functional groups and a lower degree of defects, as well as a higher degree of graphitization, which was in good agreement with the XRD and Raman results.

\subsection{Characterization of GO and rGO}

Fig. 4 shows the XRD spectra of the GO and rGO samples synthesized from the natural graphites with different degrees of graphitization. The characteristic diffraction peaks of the GO samples corresponding to the (002) plane were observed at $2 \theta=10^{\circ}$ with an average $d$-spacing of around $0.79 \mathrm{~nm}$, as shown in Fig. 4a. The results indicated that the interlayer spacing was extended due to the intercalation of the oxygen-containing functional groups into the layers, damaging the original crystals of the natural graphite samples. ${ }^{36}$ In addition, the characteristic peaks observed for the (002) reflection planes of graphite at $2 \theta=26.53^{\circ}$ decreased in intensity; its was notable that the peak observed for GO-AG had almost disappeared, while that of
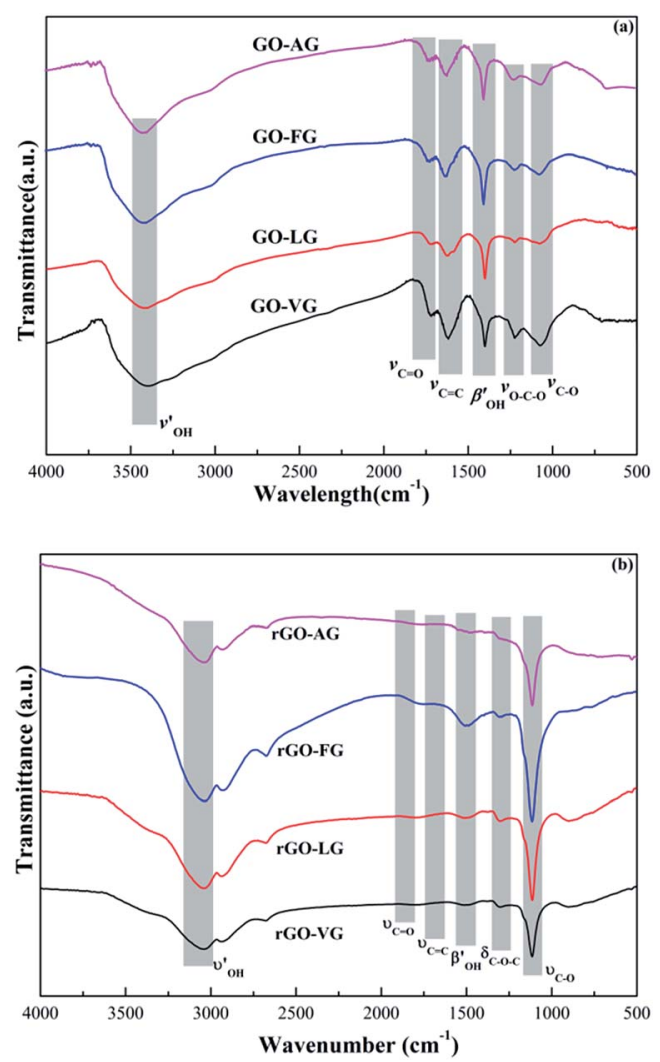

Fig. 5 FT-IR spectra obtained for the as-prepared GrOs (a) and rGOs (b).
GO-VG, GO-LG and GO-FG still existed with diminishing intensity, opposite to the increasing order observed for the degree of graphitization.

From the XRD patterns of the rGO samples displayed in Fig. $4 \mathrm{~b}$, dominant and broad peaks at approximately $2 \theta=24.5^{\circ}$ were observed with a $d$-spacing of around $0.36 \mathrm{~nm}$, which indicated a slight difference when compared to the natural graphite samples $(0.336 \mathrm{~nm})$. These findings demonstrated that the crystalline structure can be restored after the reduction process used to synthesize few-layer graphene. In addition, the $d$-spacing of the (002) plane of the rGO samples prepared from the graphites with various degrees of graphitization showed negligible differences.

The FT-IR spectra obtained for the GO and rGO samples are shown in Fig. 5. For the spectra recorded for the GO samples, distinct and strong peaks were observed at approximately 3440$3200 \mathrm{~cm}^{-1}$ as a result of the $-\mathrm{OH}$ stretching vibrations of the hydroxyl groups. ${ }^{37}$ The peaks located at around $1724 \mathrm{~cm}^{-1}$ were ascribed to the $\mathrm{C}=\mathrm{O}$ stretching vibrations of the carbonyl and carboxylic groups. ${ }^{38}$ In addition, the peaks located at around 1619 and $1400 \mathrm{~cm}^{-1}$ were attributed to the $\mathrm{C}=\mathrm{C}$ stretching mode of the non-oxidized graphitic domains and the deformation vibration of tertiary $\mathrm{C}-\mathrm{OH}$ groups, respectively. ${ }^{39}$ Moreover, the peaks centered at $1224 \mathrm{~cm}^{-1}$ were related to the stretching vibrations of $\mathrm{C}-\mathrm{O}-\mathrm{C}$ and $\mathrm{C}-\mathrm{O}$ at $1063 \mathrm{~cm}^{-1} \cdot{ }^{40} \mathrm{In}$ general, the adsorption peaks of the oxygen-containing
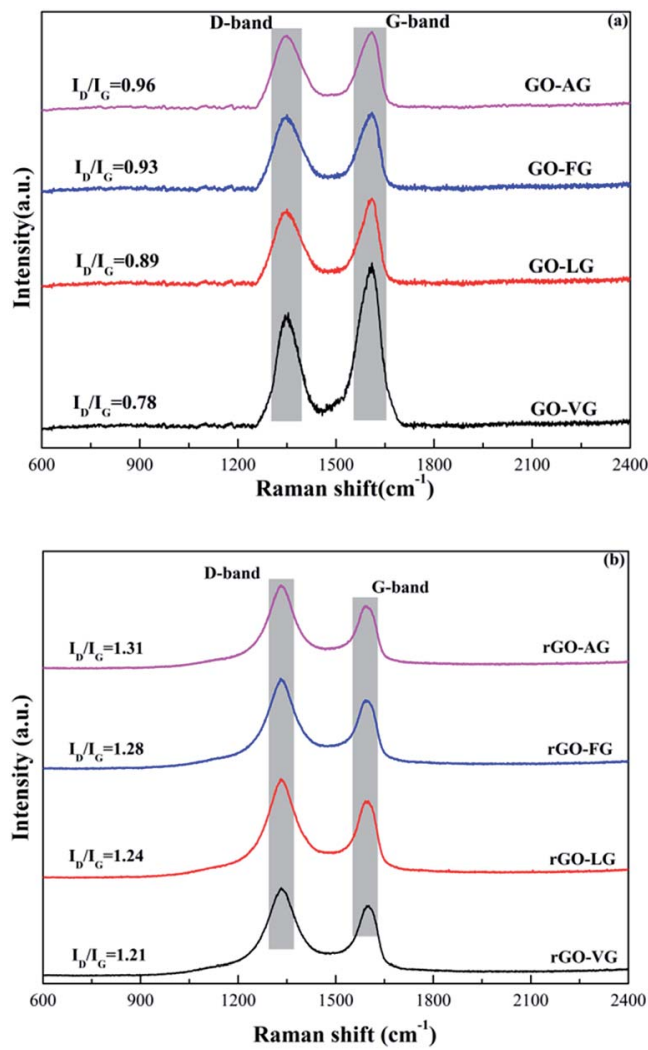

Fig. 6 Raman spectra obtained for the as-prepared GrOs (a) and rGOs (b). 
functional groups exposed on the edges were nearly the same in terms of peak location and intensity after the oxidation process.

Fig. 5b presents the FT-IR patterns of the rGO samples after the chemical reduction process. The peaks at $3440-3200 \mathrm{~cm}^{-1}$ observed for the $-\mathrm{OH}$ stretching vibrations changed slightly. In addition, the intensities of the peaks at $1724 \mathrm{~cm}^{-1}$ corresponding to the $\mathrm{C}=\mathrm{O}$ stretching vibrations, at $1400 \mathrm{~cm}^{-1}$ corresponding to $\mathrm{C}-\mathrm{OH}$ deformation vibrations and at $1224 \mathrm{~cm}^{-1}$ corresponding to the $\mathrm{C}-\mathrm{O}-\mathrm{C}$ stretching vibrations were significantly reduced. On the contrary, the intensities of the peaks at
$1063 \mathrm{~cm}^{-1}$ due to the $\mathrm{C}-\mathrm{O}$ stretching vibrations increased significantly, implying that the chemical reduction process could effectively promote the process of removing the hydroxyl and carboxyl functionalities.

The Raman spectra of the GO and rGO powders prepared from the four types of graphite are shown in Fig. 6. Two predominant and remarkable peaks corresponding to the $\mathrm{D}$ band at around $1350 \mathrm{~cm}^{-1}$ and G-band at around $1595 \mathrm{~cm}^{-1}$ were observed due to the breathing mode of the k-point phonons with $\mathrm{A}_{1 \mathrm{~g}}$ symmetry and first order scattering of the (a)
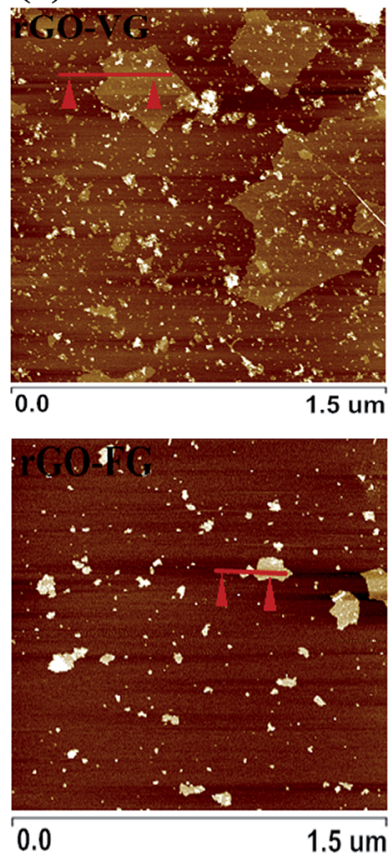

(b)

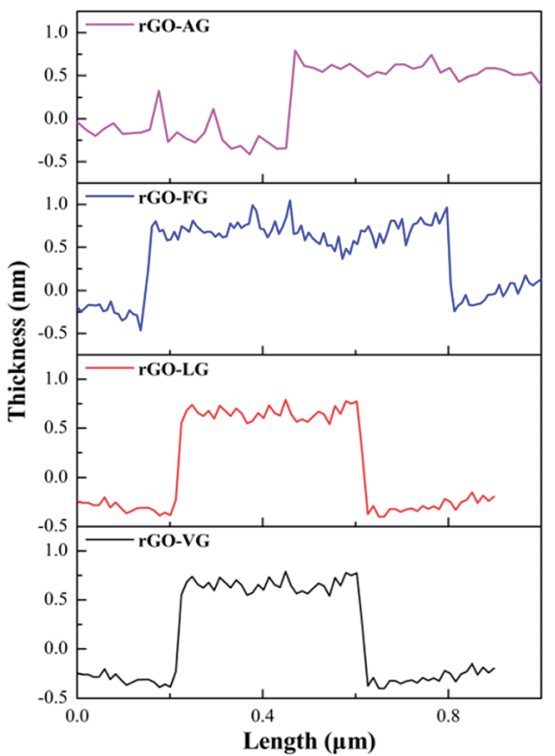

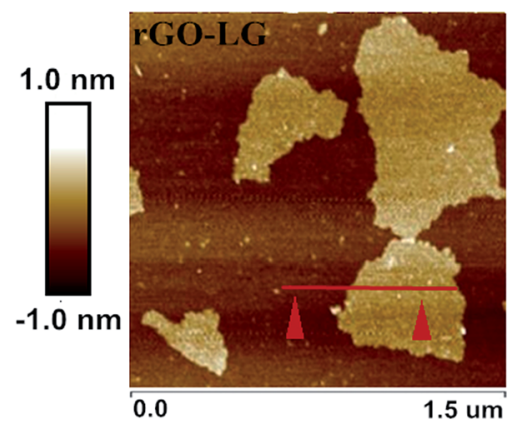
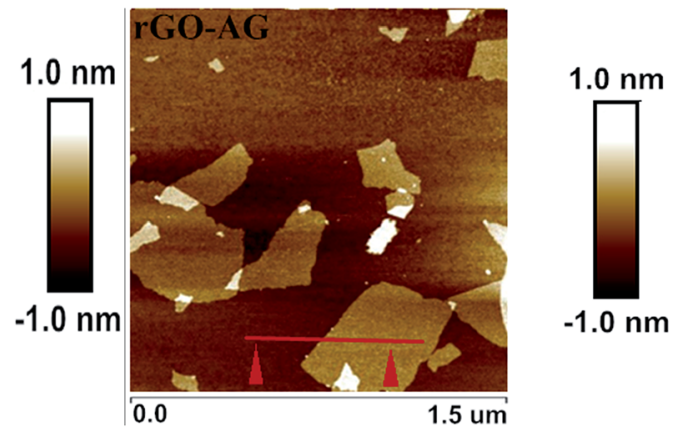

(c)

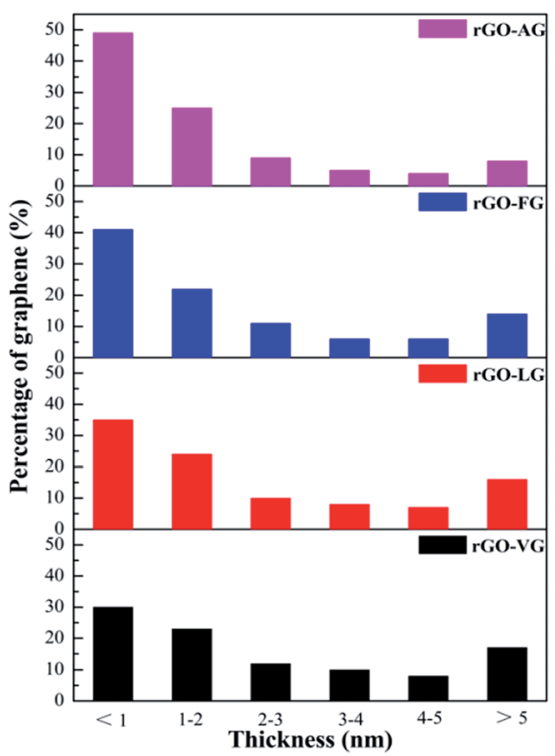

Fig. 7 AFM images (a), corresponding height profiles (b) and thickness distribution histograms (c) obtained for the as-prepared rGOs (c). 
$\mathrm{E}_{2 \mathrm{~g}}$ phonons, respectively. ${ }^{\mathbf{4 1 , 4 2}}$ On the basis of Raman analysis, the values of $I_{\mathrm{D}} / I_{\mathrm{G}}$ were calculated to be 0.78 for GO-VG, 0.89 for GO-LG, 0.93 for GO-FG and 0.96 for GO-AG. In addition, the crystallite size $\left(L_{\mathrm{a}}\right)$ was used to assess the average size of the adjacent carbon atoms in the GO and rGO samples in the contiguous region and was able to be estimated according to the following equation.

$$
L_{\mathrm{a}}=44 \times\left(I_{\mathrm{D}} / I_{\mathrm{G}}\right)^{-1}
$$

Based on the $I_{\mathrm{D}} / I_{\mathrm{G}}$ results, the corresponding values of $L_{\mathrm{a}}$ were $55.70 \mathrm{~nm}$ for GO-VG, 52.38 for GO-LG, 47.31 for GO-FG and 45.83 for GO-AG, indicating that a high degree of graphitization corresponds to a relatively low degree of defects, and the incremental order of the in-plane $\mathrm{sp}^{2}$ domains of GO-AG, GOFG, GO-LG and GO-VG was obtained. Furthermore, the defects are closely related to the degree of oxidation, meaning that the GO samples prepared from the graphites with a low degree of graphitization contained more oxygen-containing functional groups.

With regard to the Raman spectra obtained for the rGO samples, the intensities of the D-bands became stronger, while the intensities of the G-bands became weaker. The locations of the two bands displayed a small shift, implying that some of the defects were repaired during the chemical reduction process. In addition, the $I_{\mathrm{D}} / I_{\mathrm{G}}$ values were 1.21 for rGO-VG, 1.24 for rGO-LG, 1.28 for rGO-FG and 1.31 for rGO-AG, indicating that the chemical reduction process can effectively remove the oxygencontaining functional groups at the edge of the rGO samples, however, the process may also induce more defects to be formed. ${ }^{43}$ The values of $L_{\mathrm{a}}$ were calculated to be $36.36,35.48$, 34.38 and 33.59 for rGO-VG, rGO-LG, rGO-FG and rGO-AG, respectively, showing a diminishing sequence for the in-plane $\mathrm{sp}^{2}$ domains from rGO-VG to rGO-AG.

Fig. 7a and $\mathrm{b}$ present the representative AFM images and cross-section analysis along the lines in the AFM images for the individual sheets of the as-prepared rGO samples. The typical thickness obtained from the AFM images was approximately $0.781 \mathrm{~nm}$ for rGO-VG, $0.750 \mathrm{~nm}$ for rGO-LG, $0.686 \mathrm{~nm}$ for rGOFG and $0.625 \mathrm{~nm}$ for rGO-AG, which are in good agreement with that reported for rGO. ${ }^{44}$ The thickness values were slightly greater than that observed for ideal graphene, whose thickness was around $0.335 \mathrm{~nm}$, which was attributed to the presence of carboxyl and hydroxyl groups on the edges of the rGO sheets. ${ }^{45-47}$ After counting three hundred rGO sheets, the thickness distribution was obtained, as shown in Fig. 7c, with the majority of all the rGOs thicknesses measuring less than $2 \mathrm{~nm}$. Moreover, the thickness of sheets less than $1 \mathrm{~nm}$ occupied $30.16 \%$ for rGO-VG, $35.64 \%$ for rGO-LG, $41.36 \%$ for rGOFG, and $49.89 \%$ for rGO-AG, indicating that the natural graphites with a low degree of graphitization were more suitable to synthesize less-layered rGO.

Fig. 8 shows the CV curves of the as-prepared rGO samples. In general, there was little difference between the four curves, all of which seemed to form nearly rectangular and relatively symmetrical shapes. Nevertheless, weak faradaic peaks were observed, suggesting that a few redox reactions took place, which were attributed to some oxygen-containing functional groups remaining in the rGO samples. ${ }^{48}$ In addition, based on the equation, the specific capacitance values can be obtained as 107.53 $\mathrm{F} \mathrm{g}^{-1}$ for rGO-VG, 115.17 $\mathrm{F} \mathrm{g}^{-1}$ for rGO-LG, $126.98 \mathrm{~F} \mathrm{~g}^{-1}$ for rGO-FG and $139.15 \mathrm{~F} \mathrm{~g}^{-1}$ for rGO-AG, which were ascribed to the degree of redox in the rGOs impacting the defects and microstructure of the graphite samples with different degrees of graphitization.

\subsection{Mechanism}

The oxidation, exfoliation and reduction process of the asprepared rGO samples may cause the differences observed in our results, as presented in Fig. 9. It was obvious that the different degrees of graphitization on graphite can lead to the different structures, degree of defect, thickness and specific capacitance of the rGO samples through different redox and exfoliation routes on account of the analysis described above. As is known to all, graphite with a low degree of graphitization contains more active carbon atoms on the edges of the graphitic layers and the numerous carbon atoms exposed on the layers can increase the $d$-spacing and the degree of defects in the graphite starting material. Due to the fact that it was more likely for the exposed carbon atoms to be attacked during the oxidation process, graphites with lower degrees of graphitization undergo oxidation and exfoliation more easily. In addition, the greater $d$-spacing of poorly crystallized graphite results in a weak interaction between the carbon interlayers, which promotes the intercalation and diffusion of the oxidants. ${ }^{49}$ In addition, the defects that exist in the graphite structure can shorten the route for the diffusion of oxidants, enabling the process to be facile and effective. From the schematic representation shown in Fig. 9, natural graphites with a low degree of graphitization, high degree of defect and low $\mathrm{C} / \mathrm{O}$ values are able to form well-oxidized products under similar conditions with excellent exfoliation results to obtain two-layer or less graphene after reduction, which is in accordance with the characteristic (002) peaks of graphite at $2 \theta=26^{\circ}$ in the XRD patterns of GO-AG and GO-FG almost disappearing and the formation of thick layer graphene as-observed by AFM.

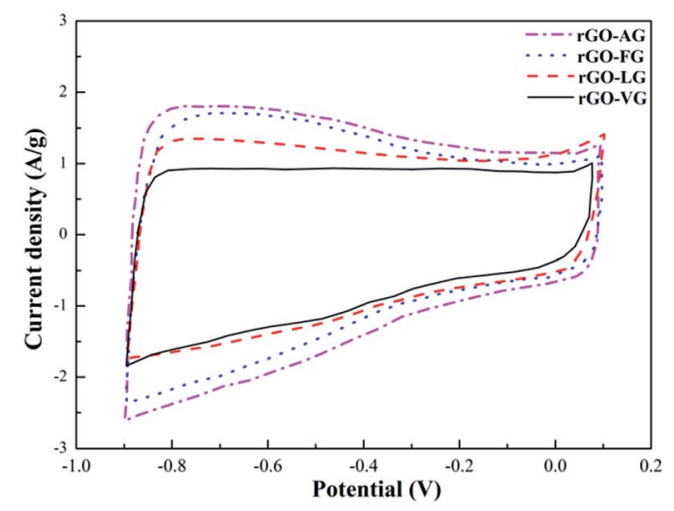

Fig. 8 CV curves obtained for the as-prepared rGOs. 

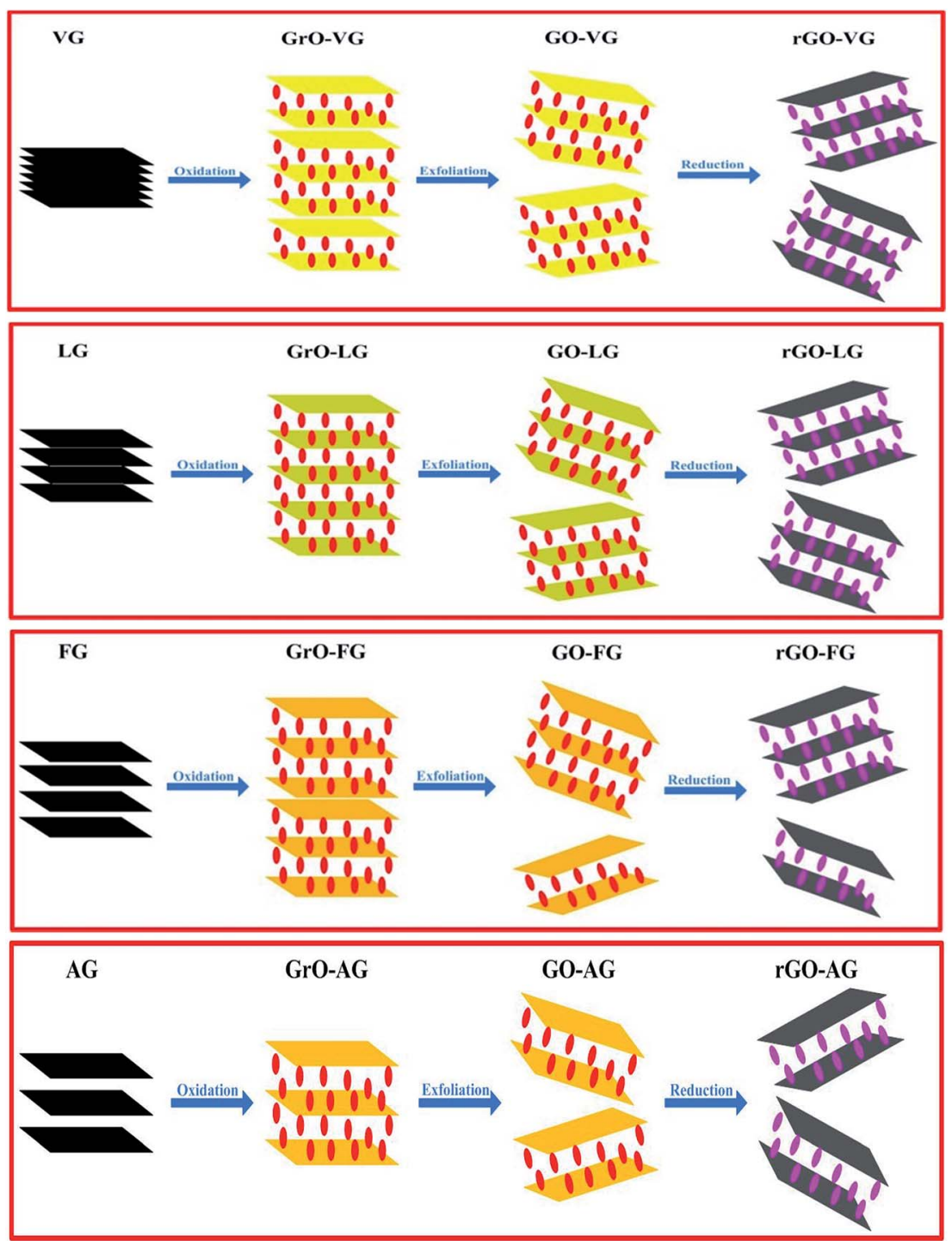

Fig. 9 Schematic process for the oxidation, exfoliation and reduction of the preparation of the rGOs.

\section{Conclusions}

Based on the characterization analysis, the results were that the degree of defects in VG, LG, FG and AG present a diminishing sequence, while the degree of graphitization and $\mathrm{C} / \mathrm{O}$ values on the surface of the four types of natural graphites increased gradually. The experimental results indicate that it was more likely for graphite with a low degree of graphitization to be sufficiently oxidized for the preparation of GO and form the thinnest layer of graphene after the reduction process. In addition, rGO synthesized using graphite with the lowest degree of graphitization has the most defects and the smallest size of in-plane $\mathrm{sp}^{2}$ domains, possessing high quality with a specific capacitance of $139.15 \mathrm{~F} \mathrm{~g}^{-1}$. The results can be attributed to the fact that most of the defects showed optimal activity and high $I_{\mathrm{D}} / I_{\mathrm{G}}$ values as well as relatively low $L_{\mathrm{a}}$ values, resulting in the small size of the in-plane $\mathrm{sp}^{2}$ domains. The smaller the size of the in-plane $\mathrm{sp}^{2}$ domains, the faster the electrons can transfer and the ion exchange taking place at the interface between the electrode and electrolyte is accelerated, resulting in the high specific capacitance observed in the electrochemical measurements. The investigations carried out in this study have established a theoretical basis to select a suitable natural graphite starting material based on the performance and characteristic properties of the graphene required for a particular application.

\section{Conflicts of interest}

There are no conflicts to declare.

\section{Acknowledgements}

The financial support for this work provided by the Fundamental Research Funds for the Central Universities (WUT: 2017zy073) is gratefully acknowledged. 


\section{References}

1 P. Solís-Fernández, R. Rozada, J. I. Paredes, S. Villar-Rodil, M. J. Fernández-Merino, L. Guardia, A. Martínez-Alonso and J. M. D. Tascón, J. Alloys Compd., 2012, 536, S532-S537.

2 W.-J. Peng, H.-Q. Li, Y.-Y. Liu and S.-X. Song, J. Mol. Liq., 2017, 230, 496-504.

3 M. Agharkar, S. Kochrekar, S. Hidouri and M. A. Azeez, Mater. Res. Bull., 2014, 59, 323-328.

4 A. K. Geim and K. S. Novoselov, Nat. Mater., 2017, 6, 183-191. 5 R. K. Joshi, S. Alwarappan, M. Yoshimura, V. Sahajwalla and Y. Nishina, Appl. Mater. Today., 2015, 1, 1-12.

6 E. P. Randviir, D. A. C. Brownson and C. E. Banks, Mater. Today, 2014, 17, 426-432.

7 S. Hermanová, M. Zarevúcká, D. Bousa, M. Mikulics and Z. Sofer, Appl. Mater. Today, 2016, 5, 200-208.

8 J.-H. Zhu, M.-J. Chen, Q.-L. He, L. Shao, S.-Y. Wei and Z.-H. Guo, RSC Adv., 2013, 3, 22790-22824.

9 H. A. Becerril, J. Mao, Z. Liu, R. M. Stoltenberg, Z. Bao and Y. Chen, ACS Nano, 2008, 2, 463-470.

10 J. Wu, W. Pisula and K. Müllen, Chem. Rev., 2007, 107, 718747.

11 C. Berger, Z. Song, X. Li, X. Wu, N. Brown, C. Naud, D. Mayou, T. Li, J. Hass and A. N. Marchenkov, Science, 2006, 312, 1191-1196.

12 L.-Y. Jiao, L. Zhang, X.-R. Wang, G. Diankov and H.-J. Dai, Nature, 2009, 458, 877-880.

13 P. W. Sutter, J. I. Flege and E. A. Sutter, Nat. Mater., 2008, 7, 406-411.

14 Z. Bo, K.-H. Yu, G.-H. Lu, P.-X. Wang, S. Mao and J.-H. Chen, Carbon, 2011, 49, 1849-1858.

15 S. Stankovich, D. A. Dikin, R. D. Piner, K. A. Kohlhaas, A. Kleinhammes, Y.-Y. Jia, Y. Wu, S. T. Nguyen and R. S. Ruoff, Carbon, 2007, 45, 1558-1565.

$16 \mathrm{Y}$. Hu, S.-X. Song and A. Lopez-Valdivieso, J. Colloid Interface Sci., 2015, 450, 68-73.

17 C. K. Chua and M. Pumera, Chem. Soc. Rev., 2014, 43, 291312.

18 A. K. Sarker and J. D. Hong, Colloids Surf., A, 2013, 436, 967974.

19 Q.-Q. Zhuo, J. Gao, M.-F. Peng, L.-L. Bai, J.-J. Deng, Y.-J. Xia, Y.-Y. Ma, J. Zhong and X.-H. Sun, Carbon, 2013, 52, 559-564.

20 W.-J. Peng, H.-Q. Li, Y. Hu, Y.-Y. Liu and S.-X. Song, Mater. Res. Bull., 2016, 74, 333-339.

21 Z.-S. Wu, W.-C. Ren, L.-C. Gao, B.-L. Liu, C.-B. Jiang and H.-M. Cheng, Carbon, 2009, 47, 493-499.

22 X. Jiao, L.-Y. Zhang, Y.-S. Qiu and J.-F. Guan, Colloids Surf., A, 2017, 529, 292-301.

23 N. Iwashita, M. Inagaki and Y. Hishiyama, Carbon, 1997, 35, 1073-1077.

24 H.-J. Yang, S. H. Yoon, Y. Korai, I. Mochida and O. Katou, Carbon, 2003, 41, 397-403.

25 S. Lim, A. S. Yoon, I. Mochida and J.-H. Chi, J. Phys. Chem. B, 2004, 108, 1533-1536.
26 C. Botas, P. Álvarez, C. Blanco, R. Santamaría, M. Granda, P. Ares, F. Rodríguez-Reinoso and R. Menéndez, Carbon, 2012, 50, 275-282.

27 C. H. A. Wong, Z. Sofer and M. Pumera, Chem.-Eur. J., 2015, 21, 8435-8440.

28 H. L. Poh, F. Sanek, A. Ambrosi, G.-J. Zhao, Z. Soferb and M. Pumera, Nanoscale, 2012, 4, 3515-3522.

29 W.-J. Peng, H.-Q. Li, Y.-Y. Liu and S.-X. Song, Appl. Surf. Sci., 2016, 364, 620-627.

30 J.-L. Liu, J.-Q. Gao, J.-K. Cheng, J.-F. Yang and G.-J. Qiao, Diamond Relat. Mater., 2006, 15, 117-120.

31 L.-H. Zou, B.-Y. Huang, Y. Huang, Q.-Z. Huang and C.-A. Wang, Mater. Chem. Phys., 2003, 82, 654-662.

32 H. Fujimoto, Carbon, 2010, 48, 3446-3453.

33 Z.-S. Wu, W.-C. Ren, L.-B. Gao, J.-P. Zhao, Z.-P. Chen, B.-L. Liu, D.-M. Tang, B. Yu, C.-B. Jiang and H.-M. Cheng, ACS Nano, 2009, 3, 411-417.

34 X. Jiao, L.-Y. Zhang, Y.-S. Qiu and Y.-R. Yuan, RSC Adv., 2017, 7, 38350-38359.

35 Y.-F. Cai, Y.-G. Pan, J.-Y. Xue, Q.-F. Sun, G.-Z. Su and X. Li, Appl. Surf. Sci., 2009, 255, 8750-8760.

36 Q.-Q. Zhuo, J. Gao, M.-F. Peng, L.-L. Bai, J.-J. Deng, Y.-J. Xia, Y.-Y. Ma, J. Zhong and X.-H. Sun, Carbon, 2013, 52, 559-564.

37 G. I. Titelman, V. Gelman, S. Bron, R. L. Khalfin, Y. Cohen and H. Bianco-Peled, Carbon, 2005, 43, 641-649.

38 T. A. Saleh, A. A. Al-Saadi and V. K. Gupta, J. Mol. Liq., 2014, 191, 85-91.

39 C. Nethravathi and M. Rajamathi, Carbon, 2008, 46, 19941998.

40 X.-W. Cui, X. Yu, L. Hou, A. Gagnoud, Y. Fautrelle, R. Moreau, Z.-M. Ren, X.-G. Lu and X. Li, J. Alloys Compd., 2016, 684, 21-28.

41 Q.-L. Huang, J.-M. Wang, W.-X. Wei, Q.-X. Yan, C.-L. Wu and X.-S. Zhu, J. Hazard. Mater., 2015, 83, 123-130.

42 S.-C. Lu, M.-G. Yao, X.-G. Yang, Q.-J. Li, J.-P. Xiao, Z. Yao, L.-H. Jiang, R. Liu, B. Liu, S.-L. Chen, B. Zou, T. Cui and B.-B. Liu, Chem. Phys. Lett., 2013, 585, 101-106.

43 F.-P. Yin, S. Wu, Y.-B. Wang, L. Wu, P.-L. Yuan and X. Wang, J. Solid State Chem., 2016, 237, 57-63.

44 P. Solís-Fernández, J. I. Paredes, S. Villar-Rodil, A. MartínezAlonso and J. M. D. Tascón, Carbon, 2010, 48, 2657-2660.

45 A. Palinkas, G. Molnar, C. Hwang, L. P. Biro and Z. Osvath, RSC Adv., 2016, 6, 86253-86258.

46 H. C. Schniepp, J. L. Li, M. J. McAllister, H. Sai, M. HerreraAlonso, D. H. Adamson, R. K. Prud'homme, R. Car, D. A. Saville and I. A. Aksay, J. Phys. Chem. B, 2006, 110, 8535-8539.

47 M. J. McAllister, J. L. Li, D. H. Adamson, H. C. Schniepp, A. A. Abdala, J. Liu, M. Herrera-Alonso, D. L. Milius, R. Car, R. K. Prud'homme and I. A. Aksay, Chem. Mater., 2007, 19, 4396-4404.

48 Z. Lin, Y. Liu, Y. Yao, O. J. Hildreth, Z. Li, K. Moon and C.-P. Wong, J. Phys. Chem. C, 2011, 115, 7120-7125.

49 G. Shao, Y. Lu, F. Wu, C. Yang, F. Zeng and Q. Wu, J. Mater. Sci., 2012, 47, 4400-4409. 